\title{
A characterization of the Gaussian Lipschitz space and sharp estimates for the Ornstein-Uhlenbeck Poisson kernel
}

\author{
Liguang Liu and Peter Sjögren
}

15 April, 2014

\begin{abstract}
The Gaussian Lipschitz space was defined by Gatto and Urbina, by means of the Ornstein-Uhlenbeck Poisson kernel. We give a characterization of this space in terms of a combination of ordinary Lipschitz continuity conditions. The main tools used in the proof are sharp estimates of the Ornstein-Uhlenbeck Poisson kernel and some of its derivatives.
\end{abstract}

\section{Introduction and main results}

Let $\gamma$ be the Gauss measure on $\mathbb{R}^{n}$ with $n \geq 1$, that is, $d \gamma(x)=\pi^{-n / 2} e^{-|x|^{2}} d x$. The Gaussian analogue of the Euclidean Laplacian is the Ornstein-Uhlenbeck operator $\mathcal{L}=-\frac{1}{2} \Delta+$ $x \cdot \nabla$, where $\nabla:=\left(\partial_{x_{1}}, \ldots, \partial_{x_{n}}\right)$. The operator $\mathcal{L}$ is the infinitesimal generator of the OrnsteinUhlenbeck semigroup $T_{t}=e^{-t \mathcal{L}}, t>0$, given by

$$
T_{t} f(x)=\pi^{-n / 2} \int_{\mathbb{R}^{n}} M_{e^{-t}}(x, y) f(y) d y
$$

for all $f \in L^{2}(\gamma)$ and $x \in \mathbb{R}^{n}$, where $M_{e^{-t}}$ is the Mehler kernel defined by

$$
M_{r}(x, y):=\frac{e^{-\frac{|y-r x|^{2}}{1-r^{2}}}}{\left(1-r^{2}\right)^{n / 2}} \quad x, y \in \mathbb{R}^{n}, \quad 0<r<1 .
$$

The Ornstein-Uhlenbeck Poisson semigroup $\left\{P_{t}\right\}_{t>0}$ is defined by subordination from $\left\{T_{t}\right\}_{t>0}$ as

$$
P_{t} f(x)=\frac{1}{\sqrt{\pi}} \int_{0}^{\infty} \frac{e^{-u}}{\sqrt{u}} T_{t^{2} /(4 u)} f(x) d u .
$$

There is a corresponding Ornstein-Uhlenbeck Poisson kernel $P_{t}(x, y)$, for which

$$
P_{t} f(x)=\int_{\mathbb{R}^{n}} P_{t}(x, y) f(y) d y
$$

2010 Mathematics Subject Classification. Primary 26A16; Secondary 28C20, 46E35.

Key words and phrases. Gauss measure space, Lipschitz space, Ornstein-Uhlenbeck Poisson kernel.

The first author is supported by National Natural Science Foundation of China (Grant No. 11101425) and the Alexander von Humboldt Foundation. 
and it is obtained from the Mehler kernel by similar subordination. Transforming variables $s=t^{2} /(4 u)$ and inserting the expression for the Mehler kernel $M_{e^{-s}}$, one gets

$$
P_{t}(x, y)=\frac{1}{2 \pi^{(n+1) / 2}} \int_{0}^{\infty} \frac{t}{s^{3 / 2}} e^{-\frac{t^{2}}{4 s}} \frac{e^{-\frac{\left|y-e^{-s} x\right|^{2}}{1-e^{-2 s}}}}{\left(1-e^{-2 s}\right)^{n / 2}} d s
$$

Gatto and Urbina [3] introduced the Gaussian Lipschitz spaces; see also [2] and [4]. Let $\alpha \in(0,1)$, which will be fixed throughout the paper. A function $f$ in $\mathbb{R}^{n}$ is said to be in the Gaussian Lipschitz space GLip if $_{\alpha}$ it is bounded and satisfies

$$
\left\|\partial_{t} P_{t} f\right\|_{L^{\infty}} \leq A t^{\alpha-1}, \quad t>0
$$

for some $A>0$. The norm in $f \in \operatorname{GLip}_{\alpha}$ is

$$
\|f\|_{\mathrm{GLip}_{\alpha}}:=\|f\|_{L^{\infty}}+\inf \{A: A \text { satisfies }(1.2)\} \text {. }
$$

The standard Euclidean Lipschitz space $\operatorname{Lip}_{\alpha}\left(\mathbb{R}^{n}\right)$ consists of all bounded functions $f$ such that for some $C>0$,

$$
|f(x)-f(y)| \leq C|x-y|^{\alpha}, \quad x, y \in \mathbb{R}^{n} .
$$

It is known that the $\operatorname{space}^{\operatorname{Lip}}\left(\mathbb{R}^{n}\right)$ can be characterized by means of the standard Poisson kernel

$$
\mathscr{P}_{t}(x, y)=c_{n} \frac{t}{\left(t^{2}+|x-y|^{2}\right)^{(n+1) / 2}}
$$

see Stein [5, Section V.4.2]. To be precise, a bounded function $f$ belongs to $\operatorname{Lip}_{\alpha}\left(\mathbb{R}^{n}\right)$ if and only if

$$
\left\|\partial_{t} \mathscr{P}_{t} f\right\|_{L^{\infty}} \leq C t^{\alpha-1}
$$

for all $t>0$. The main aim of this paper is to describe the Gaussian Lipschitz space by means of a condition like (1.3), as follows.

Theorem 1.1. Let $\alpha \in(0,1)$. The following statements are equivalent:

(i) $f \in \mathrm{GLip}_{\alpha}$;

(ii) there exists a positive constant $K$ such that for all $x, y \in \mathbb{R}^{n}$,

$$
|f(x)-f(y)| \leq K \min \left\{|x-y|^{\alpha}, \quad\left(\frac{|x-y|}{1+|x|+|y|}\right)^{\frac{\alpha}{2}}+((|x|+|y|) \sin \theta)^{\alpha}\right\}
$$

after correction of $f$ on a null set. Here $\theta$ denotes the angle between the vectors $x$ and $y$; if $x=0$ or $y=0$, then $\theta$ is understood as 0 .

Moreover, the norm $\|f\|_{\operatorname{GLip}_{\alpha}}$ is equivalent to $|f(0)|+\inf \{K>0: K$ satisfies (1.4) $\}$. 
In one dimension, the inequality (1.4) reads

$$
|f(x)-f(y)| \leq K \min \left\{|x-y|^{\alpha},\left(\frac{|x-y|}{1+|x|+|y|}\right)^{\frac{\alpha}{2}}\right\} .
$$

This is a combined Lipschitz condition, with exponent $\alpha$ for short distance $|x-y|$ (in fact, shorter than $1 /(1+|x|+|y|))$, and exponent $\alpha / 2$, with a different coefficient, for long distance. In higher dimension, the expression $(|x|+|y|) \sin \theta$ describes the "orthogonal component" of the vector $x-y$, since it is the distance from $y$ to the line in the direction $x$ plus the vice versa quantity. To make this more clear, we state an unsymmetric inequality equivalent to (1.4). For $x, y \in \mathbb{R}^{n}$ with $x \neq 0$, we decompose $y$ as $y=y_{x}+y_{x}^{\prime}$, where $y_{x}$ is parallel to $x$ and $y_{x}^{\prime}$ orthogonal to $x$. If $x=0$, we let $y_{x}=y$ and $y_{x}^{\prime}=0$, and this holds for all $x$ in case $n=1$. As proved in Lemma 2.1 below, (1.4) is equivalent to

$$
|f(x)-f(y)| \leq K^{\prime} \min \left\{|x-y|^{\alpha},\left(\frac{\left|x-y_{x}\right|}{1+|x|}\right)^{\frac{\alpha}{2}}+\left|y_{x}^{\prime}\right|^{\alpha}\right\}
$$

in any dimension, with a constant $K^{\prime}>0$ comparable with $K$. This means that the combined Lipschitz condition applies in the radial direction, but in the orthogonal direction the exponent is always $\alpha$. In the proof of Theorem 1.1, we shall use (1.5) instead of (1.4).

The proof of Theorem 1.1 relies on pointwise estimates of the Ornstein-Uhlenbeck Poisson kernel $P_{t}(x, y)$ and its derivatives, which also have independent interest. Before stating these results, we need some notation.

Throughout the paper, we shall write $C$ for various positive constants which depend only on $n$ and $\alpha$. Given any two nonnegative quantities $A$ and $B$, the notation $A \lesssim B$ stands for $A \leq C B$ (we say that $A$ is controlled by $B$ ), and $A \gtrsim B$ means $B \lesssim A$. If $B \lesssim A \lesssim B$, we write $A \simeq B$. For positive quantities $X$, we shall write

$$
\exp ^{*}(-X)
$$

meaning $\exp (-c X)$ for some constant $c=c(n, \alpha)>0$ whose value may change from one occurrence to another. Then we have for instance $t e^{-t} \simeq \exp ^{*}(-t)$ for $t>1$, since we allow different values of $c$ in the two inequalities defining the $\simeq$ relation. We shall often use inequalities like $\exp ^{*}(-X) \lesssim \exp ^{*}(-X) \exp ^{*}(-X)$.

Theorem 1.2. For all $t>0$ and $x, y \in \mathbb{R}^{n}$,

$$
P_{t}(x, y) \leq C\left[K_{1}(t, x, y)+K_{2}(t, x, y)+K_{3}(t, x, y)+K_{4}(t, x, y)\right] \text {, }
$$

where

$$
\begin{aligned}
& K_{1}(t, x, y)=\frac{t}{\left(t^{2}+|x-y|^{2}\right)^{(n+1) / 2}} \exp ^{*}(-t(1+|x|)) \\
& K_{2}(t, x, y)=\frac{t}{|x|}\left(t^{2}+\frac{\left|x-y_{x}\right|}{|x|}+\left|y_{x}^{\prime}\right|^{2}\right)^{-\frac{n+2}{2}} \exp ^{*}\left(-\frac{\left(t^{2}+\left|y_{x}^{\prime}\right|^{2}\right)|x|}{\left|x-y_{x}\right|}\right) \chi_{\left\{|x|>1, x \cdot y>0,|x| / 2 \leq\left|y_{x}\right|<|x|\right\}}
\end{aligned}
$$




$$
\begin{aligned}
& K_{3}(t, x, y)=\min (1, t) \exp ^{*}\left(-|y|^{2}\right) \\
& K_{4}(t, x, y)=\frac{t}{\left|y_{x}\right|}\left(\log \frac{|x|}{\left|y_{x}\right|}\right)^{-\frac{3}{2}} \exp ^{*}\left(-\frac{t^{2}}{\left.\log \frac{|x|}{\left|y_{x}\right|}\right)} \exp ^{*}\left(-\left|y_{x}^{\prime}\right|^{2}\right) \chi_{\left\{x \cdot y>0,1<\left|y_{x}\right|<|x| / 2\right\}} .\right.
\end{aligned}
$$

In Section 6, we consider the sharpness of Theorem 1.2. In particular, we exhibit for each of the four kernels $K_{i}(t, x, y)$ a set $\widetilde{E}_{i}$ of points $(t, x, y)$ in which $P_{t}(x, y) \simeq K_{i}(t, x, y)$ but where the other three terms $K_{j}(t, x, y)$ are much smaller; see the proof of Theorem 6.1(b). Thus none of the four terms can be suppressed in Theorem 1.2. It can also be verified that for each $i$ there exist (many) points $(t, x)$ such that the integral of $K_{i}(t, x, y)$ with respect to $y$, taken over those $y$ for which $(t, x, y) \in \widetilde{E}_{i}$, is comparable to $1=\int_{\mathbb{R}^{n}} P_{t}(x, y) d y$. This means that for these $(t, x)$, the kernel $K_{i}(t, x, \cdot)$ contains a substantial part of $P_{t}(x, \cdot)$.

We make some comments about the four terms $K_{i}$ in Theorem 1.2, focusing on large values of $|x|$.

Consider first small values of $t$. The term $K_{1}(t, x, y)$ is for $t<1 /(1+|x|)$ essentially the standard Poisson kernel. For us, the most significant term is $K_{2}(t, x, y)$, since it is the key to the term with exponent $\alpha / 2$ in (1.4) and (1.5). In one dimension,

$$
P_{t}(x, y) \lesssim K_{2}(t, x, y) \lesssim \widetilde{K}_{2}(t, x, y):=\frac{1}{t^{2}|x|}\left(1+\frac{|x-y|}{t^{2}|x|}\right)^{-\frac{3}{2}},
$$

and these estimates are sharp when $x>1$ and $3 x / 4<y<x-t^{2} x$ (see Section 6 ). Notice that $\widetilde{K}_{2}(t, x, y)$ is a Poisson-like kernel but with a dilation parameter $t^{2}|x|$ which depends on $x$, and with a slower decay as $y \rightarrow \infty$. Further, the integral in $y$ of each of the three kernels in (1.6) over the interval $\left(3 x / 4, x-t^{2} x\right)$ is of order of magnitude $1=\int_{\mathbb{R}} P_{t}(x, y) d y$. In higher dimension, $K_{2}(t, x, y)$ has, as a function of $y$, a different behavior in the $x$ direction and in the directions orthogonal to $x$.

Our Poisson kernel $P_{t}$ can be compared with the standard Poisson kernel $\mathscr{P}_{t}$ in the following way. Roughly speaking, the main part of the standard Poisson integral $\mathscr{P}_{t} f(x)$ is essentially the mean value of the function $f$ in a ball of radius $t$, centered at $x$. The analog for $P_{t} f(x)$ is the mean value in a cylinder in the $x$ direction of length $t^{2}|x|$, radius $t$ and center $x-t^{2} x$. This displacement from $x$ of the center is not very significant, since the displacement is not larger than the length.

This displacement comes from the Mehler kernel; the subordination formula says that $P_{t}$ is a weighted mean in the $t$ variable of values of the Mehler kernel. For small $t$, the Mehler kernel gives essentially the mean value of the function in a ball of radius $\sqrt{t}$ and center $e^{-t} x \approx x-t x$. So for $t<<1 /|x|^{2}$, the displacement is significant here, since it is much larger than the radius. Actually, it is only this displacement that makes the Mehler kernel essentially different from the standard heat kernel, for small $t$. Observe that the displacement is in the negative $x$ direction in both cases.

For large $t$, the Mehler kernel has a dilation factor which is essentially 1, and the displacement is to the origin. As a result, we get for $P_{t}$ the terms $K_{3}(t, x, y)$ and $K_{4}(t, x, y)$, which are large for small $y$ only.

After finishing this paper, we learned that Garrigós et al. [1, Lemmas 4.1 and 4.2] also 
estimated the kernel $P_{t}(x, y)$. Their estimates are rather different from ours and intended for other purposes.

From the proof of Theorem 1.2, it will be seen that $t \partial_{t} P_{t}$ and $t \partial_{x_{i}} P_{t}$ with $1 \leq i \leq n$ satisfy the same estimates as $P_{t}$, as follows.

Theorem 1.3. Let $i \in\{1,2, \ldots, n\}$. Then for all $t>0$ and $x, y \in \mathbb{R}^{n}$,

$$
\left|t \partial_{t} P_{t}(x, y)\right|+\left|t \partial_{x_{i}} P_{t}(x, y)\right| \leq C\left[K_{1}(t, x, y)+K_{2}(t, x, y)+K_{3}(t, x, y)+K_{4}(t, x, y)\right] .
$$

For the derivative of $P_{t}(x, y)$ with respect to $x$ in the radial direction, i. e., along the vector

$x$, we obtain a sharper estimate than that of Theorem 1.3. This result will be of fundamental importance in the proof of Theorem 1.1. To state it in a simple way, we first observe that $P_{t}$ is invariant under rotation in the sense that $P_{t}(A x, A y)=P_{t}(x, y)$ for any orthogonal matrix $A$. The same is true for all the kernels we use. This means that in our estimates, we can assume without restriction that $x=\left(x_{1}, 0, \ldots, 0\right)$ with $x_{1} \geq 0$. Then we will write the decomposition of $y$ as $y=\left(y_{1}, y^{\prime}\right) \in \mathbb{R} \times \mathbb{R}^{n-1}$.

Theorem 1.4. For all $t>0, x=\left(x_{1}, 0, \ldots, 0\right) \in \mathbb{R}^{n}$ with $x_{1} \geq 0$ and $y=\left(y_{1}, y^{\prime}\right) \in \mathbb{R}^{n}$,

$$
\left|\partial_{x_{1}} P_{t}(x, y)\right| \leq C\left[Z_{1}(t, x, y)+Z_{2}(t, x, y)+Z_{3}(t, x, y)+Z_{4}(t, x, y)\right],
$$

where

$$
\begin{aligned}
& Z_{1}(t, x, y)=\frac{t}{\left(t^{2}+|x-y|^{2}\right)^{(n+2) / 2}} \exp ^{*}(-t(1+|x|)) \\
& Z_{2}(t, x, y)=\frac{t}{x_{1}^{2}}\left(t^{2}+\frac{x_{1}-y_{1}}{x_{1}}+\left|y^{\prime}\right|^{2}\right)^{-\frac{n+4}{2}} \exp ^{*}\left(-\frac{\left(t^{2}+\left|y^{\prime}\right|^{2}\right) x_{1}}{x_{1}-y_{1}}\right) \chi_{\left\{x_{1}>1, x_{1} / 2 \leq y_{1}<x_{1}\right\}} ; \\
& Z_{3}(t, x, y)=\frac{\min \left\{t, t^{-2}\right\}}{1+|x|} \exp ^{*}\left(-|y|^{2}\right) ; \\
& Z_{4}(t, x, y)=\frac{t}{x_{1} y_{1}}\left(\log \frac{x_{1}}{y_{1}}\right)^{-\frac{5}{2}} \exp ^{*}\left(-\frac{t^{2}}{\log \frac{x_{1}}{y_{1}}}\right) \exp ^{*}\left(-\left|y^{\prime}\right|^{2}\right) \chi_{\left\{1<y_{1}<x_{1} / 2\right\}} .
\end{aligned}
$$

The paper is organized as follows. In Section 2, we prove the equivalence between the conditions (1.4) and (1.5) and then give some basic estimates needed later. Section 3 contains the proof of Theorem 1.1, assuming Theorems 1.2, 1.3 and 1.4. The proofs of Theorems 1.2 and 1.3 are given in Section 4. Section 5 contains the proof of Theorem 1.4, which is based on that of Theorem 1.2 but now exploiting also some cancellation in the integral estimates. Finally, Section 6 deals with the sharpness of our estimates for $P_{t}$.

\section{Auxiliary results}

Lemma 2.1. Let $\alpha \in(0,1)$. The conditions (1.4) and (1.5) are equivalent, and each of them implies that the function $f$ is bounded. More precisely,

$$
\sup _{x \in \mathbb{R}^{n}}|f(x)-f(0)| \lesssim \inf K \simeq \inf K^{\prime} .
$$


Proof. To see that each of the two conditions implies boundedness, it is enough to take $y=0$ in either condition. This also gives the inequality in (2.1) and the analogous inequality for (1.5).

Let $A$ and $B$ denote the minima appearing in (1.4) and (1.5), respectively. If $|x|+|y| \leq 2$, one finds that $A \simeq|x-y|^{\alpha} \simeq B$. Assume next that $|y| / 2<|x|<2|y|$. Then $\left|y_{x}^{\prime}\right| \simeq(|x|+|y|) \sin \theta$ and it is obvious that $B \lesssim A$. The converse $A \lesssim B$ is easy when $\left|y_{x}^{\prime}\right| \leq\left|x-y_{x}\right|$. When $\left|y_{x}^{\prime}\right|>\left|x-y_{x}\right|$, we have

$$
A \leq|x-y|^{\alpha} \simeq\left|y_{x}^{\prime}\right|^{\alpha} \leq B .
$$

Thus it only remains to consider the case when $|x|+|y|>2$ and $|x| /|y| \notin(1 / 2,2)$. But then $A, B \gtrsim 1$, and via the boundedness we just proved, we see that each of the inequalities (1.4) and (1.5) implies the other for these $x, y$.

Altogether, this proves the equivalence, and (2.1) also follows.

Lemma 2.2. Let $a, T, A \in(0, \infty), X \in[0, \infty)$ and $\beta \in(1, \infty)$. Then,

$$
\mathcal{J}:=\int_{0}^{a} \frac{1}{\sigma^{\beta}} \exp ^{*}\left(-\frac{T^{2}}{\sigma}\right) \exp ^{*}\left(-\frac{A^{2}}{\sigma}\right) \exp ^{*}\left(-\sigma X^{2}\right) d \sigma \leq M \frac{\exp ^{*}\left(-\frac{A T}{a}\right) \exp ^{*}(-T X)}{\left(T^{2}+A^{2}\right)^{\beta-1}},
$$

where $M>0$ is independent of $a, T, A$ and $X$.

Proof. Notice that $\exp ^{*}\left(-\frac{T^{2}}{\sigma}\right) \exp ^{*}\left(-\sigma X^{2}\right) \lesssim \exp ^{*}(-T X)$. Via a change of variable $u=$ $\left(T^{2}+A^{2}\right) / \sigma$, we see that

$$
\begin{aligned}
\mathcal{J} & \lesssim \exp ^{*}(-T X) \int_{0}^{a} \frac{1}{\sigma^{\beta}} \exp ^{*}\left(-\frac{T^{2}}{\sigma}\right) \exp ^{*}\left(-\frac{A^{2}}{\sigma}\right) d \sigma \\
& \lesssim \exp ^{*}(-T X) \frac{1}{\left(T^{2}+A^{2}\right)^{\beta-1}} \int_{\frac{T^{2}+A^{2}}{a}}^{\infty} u^{\beta-2} \exp ^{*}(-u) d u
\end{aligned}
$$

Since $\left(T^{2}+A^{2}\right) / a \geq 2 A T / a$ and $\beta>1$, the last integral is controlled by $\exp ^{*}(-A T / a)$.

Proposition 2.3. For all $x \in \mathbb{R}^{n}$ and $t>0$, the $K_{i}$ from Theorem 1.2 satisfy

$$
\int_{\mathbb{R}^{n}}\left[K_{1}(t, x, y)+K_{2}(t, x, y)\right] d y \leq C
$$

and

$$
\int_{\mathbb{R}^{n}}\left[K_{3}(t, x, y)+K_{4}(t, x, y)\right] d y \leq C \min \{1, t\} .
$$

Proof. Since $K_{1}$ is dominated by the standard Poisson kernel, it follows that $\int_{\mathbb{R}^{n}} K_{1}(t, x, y) d y \lesssim$ 1. Also, it is obvious that $\int_{\mathbb{R}^{n}} K_{3}(t, x, y) d y \lesssim \min \{1, t\}$.

For the estimates of $K_{2}$ and $K_{4}$, we can make a rotation and assume that $x=\left(x_{1}, 0, \ldots, 0\right)$ with $x_{1}>1$ and write $y=\left(y_{1}, y^{\prime}\right)$. Then

$$
\int_{\mathbb{R}^{n}} K_{2}(t, x, y) d y \lesssim \int_{\mathbb{R}} \int_{\mathbb{R}^{n-1}} \frac{t}{x_{1}}\left(t^{2}+\frac{\left|x_{1}-y_{1}\right|}{x_{1}}+\left|y^{\prime}\right|^{2}\right)^{-\frac{n+2}{2}} d y^{\prime} d y_{1}
$$


Liguang Liu and Peter Sjögren

$$
\begin{aligned}
& \lesssim \int_{\mathbb{R}} \frac{t}{x_{1}}\left(t^{2}+\frac{\left|x_{1}-y_{1}\right|}{x_{1}}\right)^{-\frac{3}{2}} d y_{1} \\
& \lesssim 1,
\end{aligned}
$$

and (2.2) is proved.

In the case of $K_{4}$, we have

$$
\begin{aligned}
\int_{\mathbb{R}^{n}} K_{4}(t, x, y) d y & \lesssim \int_{1<y_{1}<x_{1} / 2} \int_{\mathbb{R}^{n-1}} \frac{t}{y_{1}}\left(\log \frac{x_{1}}{y_{1}}\right)^{-\frac{3}{2}} \exp ^{*}\left(-\frac{t^{2}}{\log \frac{x_{1}}{y_{1}}}\right) \exp ^{*}\left(-\left|y^{\prime}\right|^{2}\right) d y^{\prime} d y_{1} \\
& \simeq \int_{1<y_{1}<x_{1} / 2} \frac{t}{y_{1}}\left(\log \frac{x_{1}}{y_{1}}\right)^{-\frac{3}{2}} \exp ^{*}\left(-\frac{t^{2}}{\log \frac{x_{1}}{y_{1}}}\right) d y_{1} \\
& \lesssim \int_{\log 2}^{\infty} \frac{t}{\sqrt{\tau}} \exp ^{*}\left(-\frac{t^{2}}{\tau}\right) \frac{d \tau}{\tau} \\
& \lesssim \min \{1, t\} .
\end{aligned}
$$

This proves $(2.3)$.

\section{Proof of Theorem 1.1}

In this section, we assume Theorems 1.2, 1.3 and 1.4 and prove Theorem 1.1. Combining Proposition 2.3 with the pointwise estimates for the $x$ derivatives of the Poisson kernel in Theorems 1.3 and 1.4, we first deduce bounds for the $L^{1}$ norms of those derivatives.

Proposition 3.1. (i) For all $i \in\{1,2, \ldots, n\}, t>0$ and $x \in \mathbb{R}^{n}$,

$$
\int_{\mathbb{R}^{n}}\left|\partial_{x_{i}} P_{t}(x, y)\right| d y \leq C t^{-1}
$$

(ii) For all $t>0$ and $x=\left(x_{1}, 0, \ldots, 0\right) \in \mathbb{R}^{n}$ with $x_{1} \geq 0$,

$$
\int_{\mathbb{R}^{n}}\left|\partial_{x_{1}} P_{t}(x, y)\right| d y \leq C t^{-2}\left(1+x_{1}\right)^{-1} .
$$

Proof. Notice that (i) follows from Theorem 1.3 and Proposition 2.3.

To prove (ii), we have, with $Z_{1}, Z_{2}, Z_{3}, Z_{4}$ as in Theorem 1.4,

$$
\left|\partial_{x_{1}} P_{t}(x, y)\right| \lesssim Z_{1}(t, x, y)+Z_{2}(t, x, y)+Z_{3}(t, x, y)+Z_{4}(t, x, y) .
$$

It is easy to see that

$$
\int_{\mathbb{R}^{n}} Z_{1}(t, x, y) d y \lesssim \int_{\mathbb{R}^{n}} \frac{t \exp ^{*}(-t(1+|x|))}{\left(t^{2}+|x-y|^{2}\right)^{(n+2) / 2}} d y \lesssim t^{-1} \exp ^{*}(-t(1+|x|)) \lesssim t^{-2}\left(1+x_{1}\right)^{-1}
$$

and

$$
\int_{\mathbb{R}^{n}} Z_{3}(t, x, y) d y \simeq \int_{\mathbb{R}^{n}} \frac{\min \left\{t, t^{-2}\right\}}{1+|x|} \exp ^{*}\left(-|y|^{2}\right) d y \lesssim \frac{\min \left\{t, t^{-2}\right\}}{1+|x|} \lesssim t^{-2}\left(1+x_{1}\right)^{-1} .
$$


Integrating $Z_{2}$ first in $y^{\prime}$ and then in $y_{1}$, we get

$$
\begin{aligned}
\int_{\mathbb{R}^{n}} Z_{2}(t, x, y) d y & \lesssim \frac{t}{x_{1}^{2}} \int_{x_{1} / 2}^{x_{1}} \int_{\mathbb{R}^{n-1}}\left(\frac{x_{1}}{x_{1}-y_{1}}\right)^{(n+4) / 2} \exp ^{*}\left(-\frac{\left(t^{2}+\left|y^{\prime}\right|^{2}\right) x_{1}}{x_{1}-y_{1}}\right) d y^{\prime} d y_{1} \\
& \lesssim \frac{t}{x_{1}^{2}} \int_{x_{1} / 2}^{x_{1}}\left(\frac{x_{1}}{x_{1}-y_{1}}\right)^{5 / 2} \exp ^{*}\left(-\frac{t^{2} x_{1}}{x_{1}-y_{1}}\right) d y_{1} \\
& \lesssim t^{-2} x_{1}^{-1}
\end{aligned}
$$

Similarly,

$$
\begin{aligned}
\int_{\mathbb{R}^{n}} Z_{4}(t, x, y) d y & \lesssim \frac{t}{x_{1}} \int_{0}^{x_{1} / 2}\left(\log \frac{x_{1}}{y_{1}}\right)^{-5 / 2} \exp ^{*}\left(-\frac{t^{2}}{\log \frac{x_{1}}{y_{1}}}\right) \frac{d y_{1}}{y_{1}} \\
& \lesssim \frac{t}{x_{1}} \int_{\log 2}^{\infty} u^{-5 / 2} \exp ^{*}\left(-\frac{t^{2}}{u}\right) d u \\
& \lesssim t^{-2} x_{1}^{-1}
\end{aligned}
$$

where $u=\log \frac{x_{1}}{y_{1}}$. Combining these estimates and noticing that $Z_{2}$ and $Z_{4}$ are non-zero only if $x_{1}>1$, we obtain (3.2).

From this proposition, we deduce two pointwise bounds for the $x$ derivatives of $P_{t} f$, with $f$ a Gaussian Lipschitz function.

Proposition 3.2. Let $\alpha \in(0,1)$ and $f \in \mathrm{GLip}_{\alpha}$ with norm 1 .

(i) For all $i \in\{1,2, \ldots, n\}, t>0$ and $x \in \mathbb{R}^{n}$,

$$
\left|\partial_{x_{i}} P_{t} f(x)\right| \leq C t^{\alpha-1} .
$$

(ii) For all $t>0$ and $x=\left(x_{1}, 0, \ldots, 0\right) \in \mathbb{R}^{n}$ with $x_{1}>0$,

$$
\left|\partial_{x_{1}} P_{t} f(x)\right| \leq C t^{\alpha-2}\left(1+x_{1}\right)^{-1} .
$$

Proof. To prove (i), we use the semigroup property of the Poisson integral and take derivatives, obtaining

$$
\partial_{x_{i}} \partial_{t} P_{s+t} f(x)=\partial_{x_{i}} \partial_{t} \int_{\mathbb{R}^{n}} P_{s}(x, y) P_{t} f(y) d y=\int_{\mathbb{R}^{n}} \partial_{x_{i}} P_{s}(x, y) \partial_{t} P_{t} f(y) d y
$$

for $s, t>0$ and $x \in \mathbb{R}^{n}$. Now let $s=t$, to get

$$
\frac{1}{2} \partial_{x_{i}} \partial_{t} P_{2 t} f(x)=\int_{\mathbb{R}^{n}} \partial_{x_{i}} P_{t}(x, y) \partial_{t} P_{t} f(y) d y .
$$

By (3.1) and the definition of $\operatorname{GLip}_{\alpha}$, this implies that for all $t>0$,

$$
\left|\partial_{x_{i}} \partial_{t} P_{t} f(x)\right| \lesssim t^{\alpha-2} .
$$


Since $f$ is bounded, it follows from (3.1) that $\partial_{x_{i}} P_{t} f(x) \rightarrow 0$ as $t \rightarrow \infty$. Thus

$$
\partial_{x_{i}} P_{t} f(x)=-\int_{t}^{\infty} \partial_{x_{i}} \partial_{\tau} P_{\tau} f(x) d \tau
$$

and (i) is a consequence of this and the preceding inequality.

We prove (ii) by a similar argument, using now (3.2). The only difference is that (3.5) is replaced by $\left|\partial_{x_{1}} \partial_{t} P_{t} f(x)\right| \lesssim t^{\alpha-3}\left(1+x_{1}\right)^{-1}$.

Proof of Theorem 1.1. To prove that (i) implies (ii), we let $f \in$ GLip $_{\alpha}$ with norm 1 and verify (1.5), using Lemma 2.1. We start by modifying $f$ on a null set. Since $f \in L^{\infty}\left(\mathbb{R}^{n}\right)$ and $\left\{P_{t}\right\}_{t>0}$ is a semigroup to which the Littlewood-Paley-Stein theory applies (see Stein [6]), we know that $P_{t} f(x) \rightarrow f(x)$ as $t \rightarrow 0$ for almost all $x \in \mathbb{R}^{n}$. For each $t>0$ and all $x \in \mathbb{R}^{n}$, one has

$$
P_{t} f(x)=P_{1} f(x)-\int_{t}^{1} \partial_{\tau} P_{\tau} f(x) d \tau,
$$

and this integral has a limit as $t \rightarrow 0$ for all $x$. We define $f(x)$ as $P_{1} f(x)-\int_{0}^{1} \partial_{\tau} P_{\tau} f(x) d \tau$ for all $x \in \mathbb{R}^{n}$.

Let $x, y \in \mathbb{R}^{n}$. For any $t>0$, one has

$$
|f(x)-f(y)| \leq\left|f(x)-P_{t} f(x)\right|+\left|P_{t} f(x)-P_{t} f(y)\right|+\left|P_{t} f(y)-f(y)\right| .
$$

Writing the first difference to the right here as an integral and applying the definition of GLip $\alpha$, we see that

$$
\left|f(x)-P_{t} f(x)\right|=\left|\int_{0}^{t} \partial_{\tau} P_{\tau} f(x) d \tau\right| \leq \int_{0}^{t} \tau^{\alpha-1} d \tau \simeq t^{\alpha}
$$

The same applies to the third difference. For the second difference, Proposition 3.2(i) yields that

$$
\left|P_{t} f(x)-P_{t} f(y)\right| \leq|x-y| \sup _{\theta \in(0,1)}\left|\nabla P_{t} f(x+\theta(y-x))\right| \lesssim|x-y| t^{\alpha-1} .
$$

Thus

$$
|f(x)-f(y)| \lesssim t^{\alpha}+|x-y| t^{\alpha-1}
$$

Taking $t=|x-y|$, we get

$$
|f(x)-f(y)| \lesssim|x-y|^{\alpha}
$$

To verify the remaining part of (ii), we first make a rotation so that $x=\left(x_{1}, 0, \ldots, 0\right)$ with $x_{1} \geq 0$. It is then enough to show that for all $y=\left(y_{1}, y^{\prime}\right) \in \mathbb{R} \times \mathbb{R}^{n-1}$,

$$
|f(x)-f(y)| \lesssim\left(\frac{\left|x_{1}-y_{1}\right|}{1+x_{1}}\right)^{\frac{\alpha}{2}}+\left|y^{\prime}\right|^{\alpha}
$$


Notice that if $|x|=x_{1} \leq 2$, then (3.8) follows directly from (3.7), since $|f|$ is bounded by 1 . If $x_{1}>2$ and $\left|x_{1}-y_{1}\right| \geq x_{1} / 2$, the right-hand side of (3.8) is greater than a positive constant, so (3.8) follows again. It only remains to consider the case $x_{1}>2$ and $\left|x_{1}-y_{1}\right|<x_{1} / 2$. For such $x$ and $y$, we write

$$
|f(x)-f(y)| \leq\left|f(x)-f\left(y_{1}, 0\right)\right|+\left|f\left(y_{1}, 0\right)-f(y)\right|,
$$

and (3.7) already implies that $\left|f\left(y_{1}, 0\right)-f(y)\right| \lesssim\left|y^{\prime}\right|^{\alpha}$. To estimate $\left|f(x)-f\left(y_{1}, 0\right)\right|$, we apply (3.6) again and proceed as before, but now using (3.4) to estimate the $x_{1}$ derivative. This gives that for any $t>0$,

$$
\left|f(x)-f\left(y_{1}, 0\right)\right| \lesssim t^{\alpha}+\left|x_{1}-y_{1}\right| t^{\alpha-2} \sup _{\theta \in[0,1]}\left|x_{1}+\theta\left(y_{1}-x_{1}\right)\right|^{-1} .
$$

Since $\left|x_{1}-y_{1}\right|<x_{1} / 2$, the supremum here is no larger than $2 x_{1}^{-1}$. Letting $t=\sqrt{\left|x_{1}-y_{1}\right| / x_{1}}$, we obtain

$$
\left|f(x)-f\left(y_{1}, 0\right)\right| \lesssim\left(\frac{\left|x_{1}-y_{1}\right|}{x_{1}}\right)^{\frac{\alpha}{2}} \simeq\left(\frac{\left|x_{1}-y_{1}\right|}{1+x_{1}}\right)^{\frac{\alpha}{2}}
$$

so (3.8) follows, and (ii) is verified.

We now prove that (ii) implies (i). Because of Lemma 2.1, we can assume that (1.5) holds with $K^{\prime} \leq 1$ and verify (1.2). Using Theorem 1.3 and the fact that $\int_{\mathbb{R}^{n}} \partial_{t} P_{t}(x, y) d y=0$, we can write

$$
\left|t \partial_{t} P_{t} f(x)\right|=\left|\int_{\mathbb{R}^{n}} t \partial_{t} P_{t}(x, y)[f(y)-f(x)] d y\right| \leq \sum_{i=1}^{4} \int_{\mathbb{R}^{n}} K_{i}(t, x, y)|f(y)-f(x)| d y .
$$

Since the condition (1.5) implies that $f \in \operatorname{Lip}_{\alpha}\left(\mathbb{R}^{n}\right)$, we have

$$
\int_{\mathbb{R}^{n}} K_{1}(t, x, y)|f(y)-f(x)| d y \lesssim \int_{\mathbb{R}^{n}} \frac{t}{(t+|x-y|)^{n+1}}|x-y|^{\alpha} d y \lesssim t^{\alpha} .
$$

From (1.5), we deduce that

$$
\begin{aligned}
& \int_{\mathbb{R}^{n}} K_{2}(t, x, y)|f(y)-f(x)| d y \\
& \quad \lesssim \int_{\mathbb{R}^{n}} \frac{t}{|x|}\left(t^{2}+\frac{\left|x-y_{x}\right|}{|x|}+\left|y_{x}^{\prime}\right|^{2}\right)^{-\frac{n+2}{2}}\left[\left(\frac{\left|x-y_{x}\right|}{1+|x|}\right)^{\frac{\alpha}{2}}+\left|y_{x}^{\prime}\right|^{\alpha}\right] d y \\
& \quad \lesssim \int_{\mathbb{R}^{n}} \frac{t}{|x|}\left(t^{2}+\frac{\left|x-y_{x}\right|}{|x|}+\left|y_{x}^{\prime}\right|^{2}\right)^{-\frac{n+2-\alpha}{2}} d y .
\end{aligned}
$$

After a rotation of coordinates, we can treat the last integral like the one in (2.4); only the exponent is different, and the resulting bound will be $C t^{\alpha}$. Finally, Proposition 2.3 implies that

$$
\begin{aligned}
\int_{\mathbb{R}^{n}}\left[K_{3}(t, x, y)+K_{4}(t, x, y)\right]|f(y)-f(x)| d y & \lesssim\|f\|_{L^{\infty}} \int_{\mathbb{R}^{n}}\left[K_{3}(t, x, y)+K_{4}(t, x, y)\right] d y \\
& \lesssim \min \{1, t\} \\
& \lesssim t^{\alpha} .
\end{aligned}
$$

We have verified (1.2). 


\section{Proof of Theorems 1.2 and 1.3}

Since $P_{t}(x, y)$ and the $K_{i}(t, x, y)$ are invariant under rotation, we only need to consider $x=\left(x_{1}, 0 \ldots, 0\right)$ with $x_{1} \geq 0$ and write $y=\left(y_{1}, y^{\prime}\right)$ as before. Theorem 1.2 is a consequence of the slightly sharper result in Proposition 4.1 below.

A change of variables $\sigma=1-e^{-s}$ in (1.1) leads to

$$
P_{t}(x, y)=\frac{1}{2 \pi^{(n+1) / 2}} \int_{0}^{1} \frac{t}{s(\sigma)^{3 / 2}} e^{-\frac{t^{2}}{4 s(\sigma)}} \frac{e^{-\frac{|y-x+\sigma x|^{2}}{1-e^{-2 s(\sigma)}}}}{\left(1-e^{-2 s(\sigma)}\right)^{n / 2}} e^{s(\sigma)} d \sigma,
$$

where $s(\sigma)=\log \frac{1}{1-\sigma}$. In the sequel, we will split the interval of integration into various subintervals, and in each subinterval we use either $s$ or $\sigma$ as variable of integration.

When $0<y_{1}<x_{1}$, the quantity

$$
\left|y-e^{-s} x\right|^{2}=|y-x+\sigma x|^{2}=\left|y_{1}-x_{1}+\sigma x_{1}\right|^{2}+\left|y^{\prime}\right|^{2}
$$

has a minimum at the point

$$
\sigma_{0}:=\frac{x_{1}-y_{1}}{x_{1}} \in(0,1)
$$

and

$$
\left|y-e^{-s} x\right|^{2}=\left(\sigma-\sigma_{0}\right)^{2} x_{1}^{2}+\left|y^{\prime}\right|^{2}, \quad 0<s<+\infty .
$$

This will be used repeatedly in what follows.

Proposition 4.1. Let $t>0, x=\left(x_{1}, 0, \ldots, 0\right) \in \mathbb{R}^{n}$ with $x_{1} \geq 0$ and $y=\left(y_{1}, y^{\prime}\right) \in \mathbb{R} \times \mathbb{R}^{n-1}$.

(i) If $y_{1} \notin\left(0, x_{1}\right)$, then

$$
P_{t}(x, y) \leq C\left[K_{1}(t, x, y)+K_{3}(t, x, y)\right]
$$

(ii) If $y_{1} \in\left[x_{1} / 2, x_{1}\right)$, then

$$
P_{t}(x, y) \leq C\left[K_{1}(t, x, y)+K_{2}(t, x, y)+K_{3}(t, x, y)\right] .
$$

(iii) If $y_{1} \in\left(0, x_{1} / 2\right)$, then

$$
P_{t}(x, y) \leq C\left[K_{1}(t, x, y)+K_{3}(t, x, y)+K_{4}(t, x, y)\right] .
$$

Proof. To prove (i), let $y_{1} \notin\left(0, x_{1}\right)$. We split the integral in (4.1) into integrals over $(0,1 / 2)$ and $[1 / 2,1)$, called $J_{1}$ and $J_{2}$.

For $J_{1}$, noticing that $\sigma \in(0,1 / 2)$ is equivalent to $s(\sigma) \in(0, \ln 2)$, we have $1-e^{-2 s(\sigma)} \simeq$ $s(\sigma) \simeq \sigma$ and $e^{s(\sigma)} \simeq 1$. As a result,

$$
J_{1} \simeq \int_{0}^{1 / 2} \frac{t}{\sigma^{(n+3) / 2}} \exp ^{*}\left(-\frac{t^{2}}{\sigma}\right) \exp ^{*}\left(-\frac{|y-x+\sigma x|^{2}}{\sigma}\right) d \sigma
$$


It follows from $y_{1} \notin\left(0, x_{1}\right)$ and $\sigma<1 / 2$ that $\left|y_{1}-x_{1}+\sigma x_{1}\right| \gtrsim \max \left\{\sigma x_{1},\left|x_{1}-y_{1}\right|\right\}$, and thus

$$
|y-x+\sigma x| \gtrsim \max \{\sigma|x|,|x-y|\} .
$$

Notice that for $\sigma \in(0,1)$, one has

$$
\exp ^{*}\left(-\frac{t^{2}}{\sigma}\right) \lesssim \exp ^{*}\left(-t^{2}\right) \lesssim \exp ^{*}(-t)
$$

Combined with Lemma 2.2, this yields that

$$
\begin{aligned}
J_{1} & \lesssim \exp ^{*}(-t) \int_{0}^{1 / 2} \frac{t}{\sigma^{(n+3) / 2}} \exp ^{*}\left(-\frac{t^{2}+|y-x|^{2}}{\sigma}\right) \exp ^{*}\left(-\sigma|x|^{2}\right) d \sigma \\
& \lesssim \frac{t}{\left(t^{2}+|y-x|^{2}\right)^{(n+1) / 2}} \exp ^{*}(-t(1+|x|)) \\
& \simeq K_{1}(t, x, y) .
\end{aligned}
$$

For $J_{2}$ we use the variable $s$, getting

$$
J_{2} \simeq \int_{\log 2}^{\infty} \frac{t}{s^{3 / 2}} \exp ^{*}\left(-\frac{t^{2}}{s}\right) \exp ^{*}\left(-\left|y-e^{-s} x\right|^{2}\right) d s
$$

Since $y_{1} \notin\left(0, x_{1}\right)$ and $s \geq \log 2$, one has $\left|y-e^{-s} x\right| \simeq\left|y_{1}-e^{-s} x_{1}\right|+\left|y^{\prime}\right| \gtrsim\left|y_{1}\right|+\left|y^{\prime}\right| \simeq|y|$ and hence

$$
\exp ^{*}\left(-\left|y-e^{-s} x\right|^{2}\right) \lesssim \exp ^{*}\left(-|y|^{2}\right)
$$

Thus,

$$
J_{2} \lesssim \exp ^{*}\left(-|y|^{2}\right) \int_{\log 2}^{\infty} \frac{t}{s^{3 / 2}} \exp ^{*}\left(-\frac{t^{2}}{s}\right) d s \lesssim \min \{1, t\} \exp ^{*}\left(-|y|^{2}\right) \simeq K_{3}(t, x, y)
$$

We have proved (4.3) and (i).

Next, we assume $y_{1} \in\left[x_{1} / 2, x_{1}\right)$ and prove (ii). With $\sigma_{0}$ given by (4.2) and now satisfying $0<\sigma_{0} \leq 1 / 2$, we split the integral in (4.1) into integrals over the three intervals $\left(0, \frac{3}{4} \sigma_{0}\right)$, $\left[\frac{3}{4} \sigma_{0}, \frac{5}{4} \sigma_{0}\right]$ and $\left(\frac{5}{4} \sigma_{0}, 1\right)$, denoted $J_{1,1}, J_{1,2}$ and $J_{1,3}$, respectively.

In $J_{1,1}$ we have $1-e^{-2 s(\sigma)} \simeq s(\sigma) \simeq \sigma$ and $e^{2 s(\sigma)} \simeq 1$, and also

$$
|y-x+\sigma x|^{2}=\left(\sigma-\sigma_{0}\right)^{2} x_{1}^{2}+\left|y^{\prime}\right|^{2} \simeq \sigma_{0}^{2} x_{1}^{2}+\left|y^{\prime}\right|^{2}=|x-y|^{2} .
$$

We get

$$
J_{1,1} \simeq \int_{0}^{3 \sigma_{0} / 4} \frac{t}{\sigma^{(n+3) / 2}} \exp ^{*}\left(-\frac{t^{2}}{\sigma}\right) \exp ^{*}\left(-\frac{|x-y|^{2}}{\sigma}\right) d \sigma
$$

Since $|x-y| \gtrsim \sigma_{0} x_{1} \geq \sigma x_{1}$, the last $\exp ^{*}$ expression here allows us to introduce also a factor $\exp ^{*}\left(-\sigma|x|^{2}\right)$ in the integrand. Because of (4.7), we can argue as in (4.8) to get $J_{1,1} \lesssim K_{1}(t, x, y)$. 
In the integral $J_{1,2}$, we have $\frac{3}{4} \sigma_{0} \leq \sigma \leq \frac{5}{4} \sigma_{0} \leq \frac{5}{8}$ and so $1-e^{-2 s(\sigma)} \simeq s(\sigma) \simeq \sigma \simeq \sigma_{0}$ and $e^{s(\sigma)} \simeq 1$. Thus,

$$
\begin{aligned}
J_{1,2} & \simeq \int_{\frac{3}{4} \sigma_{0}}^{\frac{5}{4} \sigma_{0}} \frac{t}{\sigma_{0}^{(n+3) / 2}} \exp ^{*}\left(-\frac{t^{2}}{\sigma_{0}}\right) \exp ^{*}\left(-\frac{\left(\sigma-\sigma_{0}\right)^{2} x_{1}^{2}+\left|y^{\prime}\right|^{2}}{\sigma_{0}}\right) d \sigma \\
& \simeq t\left(\frac{x_{1}}{x_{1}-y_{1}}\right)^{\frac{n+3}{2}} \exp ^{*}\left(-\frac{\left(t^{2}+\left|y^{\prime}\right|^{2}\right) x_{1}}{x_{1}-y_{1}}\right) \int_{\frac{3}{4} \sigma_{0}}^{\frac{5}{4} \sigma_{0}} \exp ^{*}\left(-\frac{x_{1}^{3}\left(\sigma-\sigma_{0}\right)^{2}}{x_{1}-y_{1}}\right) d \sigma,
\end{aligned}
$$

where we inserted the expression (4.2) for $\sigma_{0}$. The last integral, even extended to the whole line, is $\left.O\left(\left(x_{1}-y_{1}\right) / x_{1}^{3}\right)^{\frac{1}{2}}\right)$. The exp* expression preceding it is now estimated by a product of two factors. This leads to

$$
\begin{aligned}
J_{1,2} & \lesssim t\left(\frac{x_{1}}{x_{1}-y_{1}}\right)^{\frac{n+3}{2}} \min \left\{1,\left(\frac{\left(t^{2}+\left|y^{\prime}\right|^{2}\right) x_{1}}{x_{1}-y_{1}}\right)^{-\frac{n+2}{2}}\right\} \exp ^{*}\left(-\frac{\left(t^{2}+\left|y^{\prime}\right|^{2}\right) x_{1}}{x_{1}-y_{1}}\right)\left(\frac{x_{1}-y_{1}}{x_{1}^{3}}\right)^{\frac{1}{2}} \\
& \simeq K_{2}(t, x, y)
\end{aligned}
$$

for $x_{1}=|x| \geq 1$.

The last integral in (4.13) is also $O\left(\sigma_{0}\right)=O\left(\left(x_{1}-y_{1}\right) / x_{1}\right)$, and we get similarly

$$
\begin{aligned}
J_{1,2} & \lesssim t\left(\frac{x_{1}}{x_{1}-y_{1}}\right)^{\frac{n+1}{2}} \min \left\{1,\left(\frac{\left(t^{2}+\left|y^{\prime}\right|^{2}\right) x_{1}}{x_{1}-y_{1}}\right)^{-\frac{n+1}{2}}\right\} \exp ^{*}\left(-\frac{t^{2} x_{1}}{x_{1}-y_{1}}\right) \\
& \lesssim t \min \left\{\left(\frac{x_{1}}{x_{1}-y_{1}}\right)^{\frac{n+1}{2}}, \frac{1}{\left(t^{2}+\left|y^{\prime}\right|^{2}\right)^{(n+1) / 2}}\right\} \exp ^{*}\left(-t^{2}\right),
\end{aligned}
$$

where we estimated the exp* factor by means of the inequality $x_{1} /\left(x_{1}-y_{1}\right)>1$. For $x_{1}<1$, one has $x_{1}-y_{1}<1$ and so $\left(x_{1} /\left(x_{1}-y_{1}\right)\right)^{(n+1) / 2} \leq\left(x_{1}-y_{1}\right)^{-(n+1)}$, and also $\exp ^{*}\left(-t^{2}\right) \lesssim$ $\exp ^{*}(-t(1+|x|))$. As a result, $J_{1,2} \lesssim K_{1}(t, x, y)$.

To treat $J_{1,3}$, we split it into integrals over the intersection of $\left(\frac{5}{4} \sigma_{0}, 1\right)$ with each of the intervals $(0,1 / 2],(1 / 2,1)$, and denote these by $J_{1,3}^{(1)}$ and $J_{1,3}^{(2)}$, respectively.

For $J_{1,3}^{(1)}$, we may assume that $\frac{5}{4} \sigma_{0}<\frac{1}{2}$; otherwise $J_{1,3}^{(1)}=0$. Since here $\sigma \in\left(\frac{5}{4} \sigma_{0}, \frac{1}{2}\right]$, we again have $1-e^{-2 s(\sigma)} \simeq s(\sigma) \simeq \sigma$ and $e^{s(\sigma)} \simeq 1$. Further, $\left(\sigma-\sigma_{0}\right) x_{1} \simeq \sigma x_{1} \geq \sigma_{0} x_{1}=x_{1}-y_{1}$. Thus $\left(\sigma-\sigma_{0}\right) x_{1} \simeq \max \left\{\sigma x_{1}, x_{1}-y_{1}\right\}$, which implies (4.6), and the argument of (4.8) leads to $J_{1,3}^{(1)} \lesssim K_{1}(t, x, y)$.

Next, we estimate $J_{1,3}^{(2)}$. For $\max \left\{\frac{5}{4} \sigma_{0}, \frac{1}{2}\right\}<\sigma<1$, we have $\left|\sigma-\sigma_{0}\right| x_{1} \simeq \sigma x_{1} \simeq x_{1} \simeq y_{1}$ and so $|y-x+\sigma x|^{2} \gtrsim|y|^{2}$. This means that (4.10) holds and, arguing as in (4.11), we conclude that $J_{1,3}^{(2)} \lesssim K_{3}(t, x, y)$. Altogether, we obtain (4.4) and hence (ii).

Finally, we consider (iii), where $y_{1} \in\left(0, x_{1} / 2\right)$ and $\sigma_{0} \in(1 / 2,1)$. We split the integral in (4.1) into integrals over the intervals $\left(0, \sigma_{0}-\frac{y_{1}}{4 x_{1}}\right),\left[\sigma_{0}-\frac{y_{1}}{4 x_{1}}, \sigma_{0}+\frac{y_{1}}{4 x_{1}}\right]$ and $\left(\sigma_{0}+\frac{y_{1}}{4 x_{1}}, 1\right)$, and denote them by $J_{2,1}, J_{2,2}$ and $J_{2,3}$, respectively. Notice that $0<\sigma_{0}-\frac{y_{1}}{4 x_{1}}<\sigma_{0}+\frac{y_{1}}{4 x_{1}}<1$.

For $J_{2,1}$, we observe that $\sigma<\sigma_{0}-\frac{y_{1}}{4 x_{1}}$ corresponds to $s=s(\sigma)<\log \frac{x_{1}}{y_{1}}-\log \frac{5}{4}$. For such $s$ and $\sigma$, one has $e^{-s}>\frac{5 y_{1}}{4 x_{1}}$ and $|y-x+\sigma x|=\left|y-e^{-s} x\right| \simeq e^{-s} x_{1}+\left|y^{\prime}\right|$. With $s$ as variable of 
integration, we have

$$
J_{2,1} \simeq \int_{0}^{\log \frac{x_{1}}{y_{1}}-\log \frac{5}{4}} \frac{t}{s^{3 / 2}} \exp ^{*}\left(-\frac{t^{2}}{s}\right) \frac{\exp ^{*}\left(-\frac{e^{-2 s} x_{1}^{2}+\left|y^{\prime}\right|^{2}}{1-e^{-2 s}}\right)}{\left(1-e^{-2 s}\right)^{n / 2}} d s
$$

Splitting the interval of integration here by intersecting it with $(0, \log 2]$ and $(\log 2, \infty)$, we obtain two integrals denoted $J_{2,1}^{(1)}$ and $J_{2,1}^{(2)}$. For $0<s \leq \log 2$, one has $1-e^{-2 s} \simeq s \simeq \sigma$ and $e^{-s} x_{1} \simeq x_{1} \simeq x_{1}-y_{1}$. This implies (4.6) and, arguing as before, we obtain $J_{2,1}^{(1)} \lesssim K_{1}(t, x, y)$.

If $\log 2<s<\log \frac{x_{1}}{y_{1}}-\log \frac{5}{4}$, then $1-e^{-s} \simeq 1$ and $e^{-s} x_{1} \gtrsim y_{1}$, which implies (4.10) and then also $J_{2,1}^{(2)} \lesssim K_{3}$, as before. We have proved that $J_{2,1} \lesssim K_{1}+K_{3}$.

For $J_{2,2}$, we integrate in $s$, getting

$$
J_{2,2} \simeq \int_{\log \frac{x_{1}}{y_{1}}-\log \frac{5}{4}}^{\log \frac{x_{1}}{y_{1}}+\log \frac{4}{3}} \frac{t}{s^{3 / 2}} \exp ^{*}\left(-\frac{t^{2}}{s}\right) \frac{\exp \left(-\frac{\left|y-e^{-s} x\right|^{2}}{1-e^{-2 s}}\right)}{\left(1-e^{-2 s}\right)^{n / 2}} d s .
$$

Since now $x_{1}>2 y_{1}$, we see that $\log \frac{x_{1}}{y_{1}}-\log \frac{5}{4} \gtrsim 1$, which implies that $s \simeq \log \frac{x_{1}}{y_{1}}$ and $1-e^{-2 s} \simeq 1$ in this integral. Let $\tau=\log \frac{x_{1}}{y_{1}}-s$, so that $-\log \frac{4}{3} \leq \tau \leq \log \frac{5}{4}$ and

$$
\left|y-e^{-s} x\right| \simeq\left|y_{1}-e^{-s} x_{1}\right|+\left|y^{\prime}\right|=\left|\left(1-e^{\tau}\right) y_{1}\right|+\left|y^{\prime}\right| \simeq|\tau| y_{1}+\left|y^{\prime}\right| .
$$

It follows that

$$
\begin{aligned}
J_{2,2} & \simeq \frac{t}{\left(\log \frac{x_{1}}{y_{1}}\right)^{3 / 2}} \exp ^{*}\left(-\frac{t^{2}}{\log \frac{x_{1}}{y_{1}}}\right) \exp ^{*}\left(-\left|y^{\prime}\right|^{2}\right) \int_{-\log \frac{4}{3}}^{\log \frac{5}{4}} \exp ^{*}\left(-\tau^{2} y_{1}^{2}\right) d \tau \\
& \lesssim \frac{t}{\left(\log \frac{x_{1}}{y_{1}}\right)^{3 / 2}} \exp ^{*}\left(-\frac{t^{2}}{\log \frac{x_{1}}{y_{1}}}\right) \exp ^{*}\left(-\left|y^{\prime}\right|^{2}\right) \frac{1}{y_{1}} \\
& \simeq K_{4}(t, x, y)
\end{aligned}
$$

when $y_{1}>1$. If $y_{1} \in(0,1]$, we control the integral in (4.15) by 1 and obtain

$$
J_{2,2} \simeq \frac{t}{\left(\log \frac{x_{1}}{y_{1}}\right)^{3 / 2}} \exp ^{*}\left(-\frac{t^{2}}{\log \frac{x_{1}}{y_{1}}}\right) \exp ^{*}\left(-\left|y^{\prime}\right|^{2}\right) \lesssim \min \{1, t\} \exp ^{*}\left(-\left|y^{\prime}\right|^{2}\right) \simeq K_{3}(t, x, y)
$$

In $J_{2,3}$, we have $s>\log \frac{x_{1}}{y_{1}}+\log \frac{4}{3}>\log 2$ and thus $y_{1}-e^{-s} x_{1} \simeq y_{1}$, which once more leads to $(4.10)$ and $J_{2,3} \lesssim K_{3}$.

Summing up, we obtain (4.5) and (iii).

Proof of Theorem 1.3. By differentiating with respect to $t$ in (1.1), we have

$$
t \partial_{t} P_{t}(x, y)=\frac{1}{2 \pi^{(n+1) / 2}} \int_{0}^{\infty} \frac{t}{s^{3 / 2}} e^{-\frac{t^{2}}{4 s}}\left(1-\frac{t^{2}}{2 s}\right) \frac{e^{-\frac{\left|y-e^{-s} x\right|^{2}}{1-e^{-2 s}}}}{\left(1-e^{-2 s}\right)^{n / 2}} d s
$$


This expression is similar to that in (1.1), only with an extra factor $1-t^{2} / 2 s$. Since

$$
\left|1-\frac{t^{2}}{2 s}\right| e^{-\frac{t^{2}}{4 s}} \lesssim \exp ^{*}\left(-\frac{t^{2}}{s}\right)
$$

we see that all our estimates for $P_{t}$ in Proposition 4.1 remain valid for $\left|t \partial_{t} P_{t}\right|$.

For $i \in\{1,2, \ldots, n\}$, we have

$$
t \partial_{x_{i}} P_{t}(x, y)=\frac{1}{\pi^{(n+1) / 2}} \int_{0}^{\infty} \frac{t}{s^{3 / 2}} e^{-\frac{t^{2}}{4 s}} \frac{t e^{-s}\left(y_{i}-e^{-s} x_{i}\right)}{1-e^{-2 s}} \frac{e^{-\frac{\left|y-e^{-s} x\right|^{2}}{1-e^{-2 s}}}}{\left(1-e^{-2 s}\right)^{n / 2}} d s
$$

Compared with (1.1), the integrand here has an extra factor

$$
\frac{t e^{-s}\left(y_{i}-e^{-s} x_{i}\right)}{1-e^{-2 s}}=\frac{t}{\sqrt{s}} \frac{\sqrt{s} e^{-s}}{\sqrt{1-e^{-2 s}}} \frac{y_{i}-e^{-s} x_{i}}{\sqrt{1-e^{-2 s}}}
$$

Since the middle factor to the right here is bounded, we can suppress the extra factor if we replace $e^{-\frac{t^{2}}{4 s}} e^{-\frac{\left|y-e^{-s} x\right|^{2}}{1-e^{-2 s}}}$ by $\exp ^{*}\left(-\frac{t^{2}}{s}\right) \exp ^{*}\left(-\frac{\left|y-e^{-s} x\right|^{2}}{1-e^{-2 s}}\right)$ in the integral. Thus

$$
\left|t \partial_{x_{i}} P_{t}(x, y)\right| \lesssim \int_{0}^{\infty} \frac{t}{s^{3 / 2}} \exp ^{*}\left(-\frac{t^{2}}{s}\right) \frac{\exp ^{*}\left(-\frac{\left|y-e^{-s}\right|^{2}}{1-e^{-2 s}}\right)}{\left(1-e^{-2 s}\right)^{n / 2}} d s
$$

so the estimates for $P_{t}$ are valid also for $\left|t \partial_{x_{i}} P_{t}\right|$.

\section{$5 \quad$ Proof of Theorem 1.4}

Notice that

$$
\partial_{x_{1}} P_{t}(x, y)=\frac{1}{\pi^{(n+1) / 2}} \int_{0}^{\infty} \frac{t e^{-\frac{t^{2}}{4 s}}}{s^{3 / 2}} \frac{e^{-s}}{\sqrt{1-e^{-2 s}}} \frac{y_{1}-e^{-s} x_{1}}{\sqrt{1-e^{-2 s}}} \frac{e^{-\frac{\left|y-e^{-s} x\right|^{2}}{1-e^{-2 s}}}}{\left(1-e^{-2 s}\right)^{n / 2}} d s .
$$

We consider the same cases (i), (ii) (iii) as in Proposition 4.1, and exactly as in the proof of that proposition, we split the integral into parts by splitting the interval of integration. The parts will again be denoted by $J_{1}, J_{2}, J_{2,1}^{(1)}$ etc. For all these parts except $J_{1,2}$ and $J_{2,2}$, we follow closely the arguments in Section 4; in particular we often use $\sigma=1-e^{-s}$ instead of $s$.

Since

$$
\frac{\left|y-e^{-s} x\right|}{\sqrt{1-e^{-2 s}}} e^{-\frac{\left|y-e^{-s} x\right|^{2}}{1-e^{-2 s}}} \lesssim \exp ^{*}\left(-\frac{\left|y-e^{-s} x\right|^{2}}{1-e^{-2 s}}\right),
$$

the absolute value of the integrand in (5.1) is controlled by

$$
\frac{t e^{-\frac{t^{2}}{4 s}}}{s^{3 / 2}} \frac{e^{-s}}{\sqrt{1-e^{-2 s}}} \frac{\exp ^{*}\left(-\frac{\left|y-e^{-s} x\right|^{2}}{1-e^{-2 s}}\right)}{\left(1-e^{-2 s}\right)^{n / 2}}
$$


Switching to integration with respect to $\sigma$, we get instead, since $d \sigma=e^{-s} d s$,

$$
\frac{t e^{-\frac{t^{2}}{4 s}}}{s^{3 / 2}} \frac{1}{\sqrt{1-e^{-2 s}}} \frac{\exp ^{*}\left(-\frac{|y-x+\sigma x|^{2}}{1-e^{-2 s}}\right)}{\left(1-e^{-2 s}\right)^{n / 2}}
$$

where $s=s(\sigma)=\log 1 /(1-\sigma)$. Compared with the integral treated in the proof of Proposition 1.4, we now have an extra factor which for $s<\log 2$, i.e., $\sigma<1 / 2$, is controlled by $s^{-1 / 2} \simeq \sigma^{-1 / 2}$, and for $s>\log 2$ by $e^{-s}$.

For the integrals $J_{1}, J_{1,1}, J_{1,3}^{(1)}$ and $J_{2,1}^{(1)}$, we integrate in $\sigma$ and argue as in Section 4 . Because of the extra factor $\sigma^{-1 / 2}$, the exponent $(n+3) / 2$ of $\sigma$ in (5.1) will now be $(n+4) / 2$ in the analogous estimates. As a result, the bound obtained will be $Z_{1}(t, x, y)$ instead of $K_{1}(t, x, y)$.

For $J_{2}, J_{1,3}^{(2)}$ and $J_{2,1}^{(2)}$ and $J_{2,3}$, we use $s$ as variable of integration. Arguments similar to those in Section 4 show that the integrand is now dominated by

$$
\frac{t}{s^{3 / 2}} e^{-\frac{t^{2}}{4 s}} e^{-s} \exp ^{*}\left(-\left|y_{1}-e^{-s} x_{1}\right|^{2}\right) \exp ^{*}\left(-|y|^{2}\right) .
$$

The interval of integration is $(\log 2,+\infty)$ or a subset of it. Since

$$
\frac{t}{s^{3 / 2}} \exp ^{*}\left(-\frac{t^{2}}{s}\right) \lesssim \min \left\{t, t^{-2}\right\}
$$

the integrals considered are controlled by

$$
\begin{aligned}
& \min \left\{t, t^{-2}\right\} \exp ^{*}\left(-|y|^{2}\right) \int_{\log 2}^{\infty} e^{-s} \exp ^{*}\left(-\left|y_{1}-e^{-s} x_{1}\right|^{2}\right) d s \\
& \quad \lesssim \min \left\{t, t^{-2}\right\} \exp ^{*}\left(-|y|^{2}\right) \min \left\{1, \frac{1}{x_{1}}\right\} \\
& \quad \lesssim Z_{3}(t, x, y) .
\end{aligned}
$$

It remains to estimate $J_{1,2}$ and $J_{2,2}$, in which $y_{1}$ is as in (ii) and (iii) of Proposition 4.1, respectively.

For $J_{1,2}$, we thus assume $y_{1} \in\left[x_{1} / 2, x_{1}\right)$. When $0 \leq x_{1} \leq 1$, we can estimate $\left|J_{1,2}\right|$ as in (4.14). But now the four exponents $(n+1) / 2$ will be replaced by $(n+2) / 2$, and in the next step, we estimate $\left(x_{1} /\left(x_{1}-y_{1}\right)\right)^{(n+2) / 2}$ by $\left(x_{1}-y_{1}\right)^{-(n+2)}$. The result will be $\left|J_{1,2}\right| \lesssim Z_{1}(t, x, y)$.

When $x_{1}>1$, we shall estimate

$$
J_{1,2}=C \int_{\left|\sigma-\sigma_{0}\right| \leq \frac{1}{4} \sigma_{0}} \frac{t}{s(\sigma)^{3 / 2}} \exp \left(-\frac{t^{2}}{4 s(\sigma)}\right) \frac{\left(\sigma-\sigma_{0}\right) x_{1}}{1-e^{-2 s(\sigma)}} \frac{e^{-\frac{\left|\sigma-\sigma_{0}\right|^{2}|x|^{2}+\left|y^{\prime}\right|^{2}}{1-e^{-2 s(\sigma)}}}}{\left(1-e^{-2 s(\sigma)}\right)^{n / 2}} d \sigma .
$$

Here $\sigma_{0} \leq 1 / 2$, and $s(\sigma) \simeq \sigma \leq 5 / 8$ in the integral. Let us make a change of variable $u=$ $\left(\sigma-\sigma_{0}\right) x_{1}$. Then $\sigma=\sigma(u)=\sigma_{0}+u / x_{1}$, and we write $s(u)$ for $s(\sigma(u))$ so that

$$
s(u)=\log \frac{1}{1-\sigma(u)}=\log \frac{1}{1-\sigma_{0}-u / x_{1}}=\log \frac{x_{1}}{y_{1}-u} .
$$


Thus

$$
\begin{aligned}
J_{1,2} & =\frac{C}{x_{1}} \int_{|u| \leq \frac{x_{1}-y_{1}}{4}} \frac{t}{s(u)^{3 / 2}} \exp \left(-\frac{t^{2}}{4 s(u)}\right) \frac{u}{1-e^{-2 s(u)}} \frac{e^{-\frac{u^{2}+\left.y^{\prime}\right|^{2}}{1-e^{-2 s(u)}}}}{\left(1-e^{-2 s(u)}\right)^{n / 2}} d u \\
& =\frac{C}{x_{1}} \int_{|u| \leq \frac{x_{1}-y_{1}}{4}} u F(s(u), u) d u,
\end{aligned}
$$

where for $\tau \in(0, \infty)$ and $w \in \mathbb{R}$,

$$
F(\tau, w)=\frac{t}{\tau^{3 / 2}\left[1-e^{-2 \tau}\right]^{(n+2) / 2}} \exp \left(-\frac{t^{2}}{4 \tau}\right) \exp \left(-\frac{w^{2}+\left|y^{\prime}\right|^{2}}{1-e^{-2 \tau}}\right) .
$$

Notice that $F(\cdot, w)=F(\cdot,-w)$ for $w \in \mathbb{R}$. We can write

$$
J_{1,2}=\frac{C}{x_{1}} \int_{0}^{\frac{x_{1}-y_{1}}{4}} u[F(s(u), u)-F(s(-u), u)] d u,
$$

and here

$$
|F(s(u), u)-F(s(-u), u)| \leq|s(u)-s(-u)| \sup _{s(-u)<\tau<s(u)}\left|\partial_{\tau} F(\tau, u)\right| .
$$

From (5.2) and the mean value theorem, we deduce that for $0<u \leq\left(x_{1}-y_{1}\right) / 4$

$$
|s(u)-s(-u)| \leq 2 u \sup _{-u<v<u} \frac{1}{y_{1}-v} .
$$

With $s(-u)<\tau<s(u)$, we have

$$
\partial_{\tau} F(\tau, u)=F(\tau, u)\left[-\frac{3}{2 \tau}-\frac{(n+2) e^{-2 \tau}}{1-e^{-2 \tau}}+\frac{t^{2}}{4 \tau^{2}}+\frac{2\left(u^{2}+\left|y^{\prime}\right|^{2}\right) e^{-2 \tau}}{\left(1-e^{-2 \tau}\right)^{2}}\right] .
$$

Here $(n+2) e^{-2 \tau} /\left(1-e^{-2 \tau}\right) \lesssim \tau^{-1}$, and $\frac{t^{2}}{4 \tau^{2}} \exp \left(-\frac{t^{2}}{4 \tau}\right) \lesssim \tau^{-1} \exp ^{*}\left(-\frac{t^{2}}{\tau}\right)$. Further,

$$
\begin{aligned}
\frac{\left(u^{2}+\left|y^{\prime}\right|^{2}\right) e^{-2 \tau}}{\left(1-e^{-2 \tau}\right)^{2}} \exp \left(-\frac{u^{2}+\left|y^{\prime}\right|^{2}}{1-e^{-2 \tau}}\right) & \lesssim \frac{e^{-2 \tau}}{1-e^{-2 \tau}} \exp ^{*}\left(-\frac{u^{2}+\left|y^{\prime}\right|^{2}}{1-e^{-2 \tau}}\right) \\
& \lesssim \frac{1}{\tau} \exp ^{*}\left(-\frac{u^{2}+\left|y^{\prime}\right|^{2}}{1-e^{-2 \tau}}\right)
\end{aligned}
$$

and so

$$
\left|\partial_{\tau} F(\tau, u)\right| \lesssim \frac{t}{\tau^{5 / 2}\left[1-e^{-2 \tau}\right]^{(n+2) / 2}} \exp ^{*}\left(-\frac{t^{2}}{\tau}\right) \exp ^{*}\left(-\frac{u^{2}+\left|y^{\prime}\right|^{2}}{1-e^{-2 \tau}}\right) .
$$

Recall that $x_{1} / 2 \leq y_{1}<x_{1}$. In (5.5) we have $|v|<u \leq\left(x_{1}-y_{1}\right) / 4<y_{1} / 2$ so that $y_{1}-v \simeq y_{1} \simeq x_{1}$, and we conclude that

$$
|s(u)-s(-u)| \lesssim \frac{u}{x_{1}}
$$


Since all occurring values of $s( \pm u)$ and $\tau$ satisfy $s( \pm u) \simeq \tau \simeq \sigma_{0}$, (5.6) implies

$$
\sup _{s(-u)<\tau<s(u)}\left|\partial_{\tau} F(\tau, u)\right| \lesssim \frac{t}{\sigma_{0}^{(n+7) / 2}} \exp ^{*}\left(-\frac{t^{2}}{\sigma_{0}}\right) \exp ^{*}\left(-\frac{u^{2}+\left|y^{\prime}\right|^{2}}{\sigma_{0}}\right) .
$$

Inserting the last two estimates in (5.4), we obtain

$$
|F(s(u), u)-F(s(-u), u)| \lesssim \frac{u}{x_{1}} \frac{t}{\sigma_{0}^{(n+7) / 2}} \exp ^{*}\left(-\frac{t^{2}}{\sigma_{0}}\right) \exp ^{*}\left(-\frac{u^{2}+\left|y^{\prime}\right|^{2}}{\sigma_{0}}\right),
$$

which combined with (5.3) implies that

$$
\begin{aligned}
\left|J_{1,2}\right| & \lesssim \frac{t}{x_{1}^{2} \sigma_{0}^{(n+7) / 2}} \exp ^{*}\left(-\frac{t^{2}}{\sigma_{0}}\right) \int_{0}^{\frac{x_{1}-y_{1}}{4}} u^{2} \exp ^{*}\left(-\frac{u^{2}+\left|y^{\prime}\right|^{2}}{\sigma_{0}}\right) d u \\
& \lesssim \frac{t}{x_{1}^{2}} \frac{1}{\sigma_{0}^{(n+4) / 2}} \exp ^{*}\left(-\frac{t^{2}+\left|y^{\prime}\right|^{2}}{\sigma_{0}}\right) \\
& \lesssim \frac{t}{x_{1}^{2}} \frac{1}{\sigma_{0}^{(n+4) / 2}} \min \left\{1,\left(\frac{\sigma_{0}}{t^{2}+\left|y^{\prime}\right|^{2}}\right)^{(n+4) / 2}\right\} \exp ^{*}\left(-\frac{t^{2}+\left|y^{\prime}\right|^{2}}{\sigma_{0}}\right)
\end{aligned}
$$

Since $\sigma_{0}=\left(x_{1}-y_{1}\right) / x_{1}$, we see that the last expression amounts to $Z_{2}(t, x, y)$.

We shall finally estimate $J_{2,2}$, in which $y_{1} \in\left(0, x_{1} / 2\right)$. When $0<y_{1} \leq 1$, we have an upper estimate for $\left|J_{2,2}\right|$ like (4.16), but now with an extra factor $\exp \left(-\log \frac{x_{1}}{y_{1}}\right) \lesssim\left(1+x_{1}\right)^{-1}$ coming from $e^{-s}$; recall that $\left|s-\frac{x_{1}}{y_{1}}\right| \lesssim 1$. Thus

$$
\left|J_{2,2}\right| \lesssim \frac{t}{\left(\log \frac{x_{1}}{y_{1}}\right)^{3 / 2}} \exp ^{*}\left(-\frac{t^{2}}{\log \frac{x_{1}}{y_{1}}}\right) \frac{1}{1+x_{1}} \exp ^{*}\left(-\left|y^{\prime}\right|^{2}\right)
$$

The first $\exp ^{*}$ factor here is controlled by $\min \left\{1,\left(\frac{t^{2}}{\log \left(x_{1} / y_{1}\right)}\right)^{-3 / 2}\right\}$. Since $\log \frac{x_{1}}{y_{1}} \gtrsim 1$, this is seen to lead to $\left|J_{2,2}\right| \lesssim Z_{3}(t, x, y)$.

When $y_{1}>1$, we estimate $J_{2,2}$ by modifying the preceding argument for $J_{1,2}$. Instead of (5.3), we get now

$$
J_{2,2}=\frac{C}{x_{1}} \int_{0}^{\frac{y_{1}}{4}} u[F(s(u), u)-F(s(-u), u)] d u,
$$

and we still have (5.2), (5.4), (5.5) and (5.6). Since now $0 \leq u \leq y_{1} / 4$ in (5.5), it follows that $y_{1}-v \simeq y_{1}$ for any $|v|<u$, and thus

$$
|s(u)-s(-u)| \lesssim \frac{u}{y_{1}}
$$

In the estimate for $J_{2,2}$ in Section 4 , we saw that $s \simeq \log \frac{x_{1}}{y_{1}}$, which now means that $s(u) \simeq$ $s(-u) \simeq \log \frac{x_{1}}{y_{1}}$, and $\log \frac{x_{1}}{y_{1}}>\log 2$. In (5.6), we thus have $\tau \simeq \log \frac{x_{1}}{y_{1}}$ so that $1-e^{-2 \tau} \simeq 1$, which implies that

$$
\sup _{s(-u)<\tau<s(u)}\left|\partial_{\tau} F(\tau, u)\right| \lesssim t\left(\log \frac{x_{1}}{y_{1}}\right)^{-5 / 2} \exp ^{*}\left(-\frac{t^{2}}{\log \frac{x_{1}}{y_{1}}}\right) \exp ^{*}\left(-u^{2}-\left|y^{\prime}\right|^{2}\right) .
$$


Inserting the last two estimate in (5.4), we see that

$$
|F(s(u), u)-F(s(-u), u)| \lesssim \frac{t u}{y_{1}}\left(\log \frac{x_{1}}{y_{1}}\right)^{-5 / 2} \exp ^{*}\left(-\frac{t^{2}}{\log \frac{x_{1}}{y_{1}}}\right) \exp ^{*}\left(-u^{2}-\left|y^{\prime}\right|^{2}\right),
$$

which combined with (5.7) implies that

$$
\begin{aligned}
\left|J_{2,2}\right| & \lesssim \frac{t}{x_{1} y_{1}}\left(\log \frac{x_{1}}{y_{1}}\right)^{-5 / 2} \exp ^{*}\left(-\frac{t^{2}}{\log \frac{x_{1}}{y_{1}}}\right) \exp ^{*}\left(-\left|y^{\prime}\right|^{2}\right) \int_{0}^{\frac{y_{1}}{4}} u^{2} \exp ^{*}\left(-u^{2}\right) d u \\
& \lesssim \frac{t}{x_{1} y_{1}}\left(\log \frac{x_{1}}{y_{1}}\right)^{-5 / 2} \exp ^{*}\left(-\frac{t^{2}}{\log \frac{x_{1}}{y_{1}}}\right) \exp ^{*}\left(-\left|y^{\prime}\right|^{2}\right) \\
& \simeq Z_{4}(t, x, y) .
\end{aligned}
$$

Theorem 1.4 is proved.

\section{Sharpness arguments}

We let $K_{j}(t, x, y), j=1,2,3,4$, be as in Theorem 1.2. Let $\mathbb{R}_{+}=(0, \infty)$.

Theorem 6.1. (a) The estimate $P_{t}(x, y) \simeq K_{1}(t, x, y)$ holds uniformly in the set

$$
\begin{aligned}
E_{1}= & \left\{(t, x, y) \in \mathbb{R}_{+} \times \mathbb{R}^{n} \times \mathbb{R}^{n}:\right. \\
& \left.|x|>1, \quad x \cdot y>0, \quad t^{2}|x|<|x|-\left|y_{x}\right|<\frac{1}{4|x|}, \quad\left|y_{x}^{\prime}\right|<|x|-\left|y_{x}\right|\right\} .
\end{aligned}
$$

Similarly, $P_{t}(x, y) \simeq K_{2}(t, x, y)$ uniformly in

$$
\begin{aligned}
E_{2}= & \left\{(t, x, y) \in \mathbb{R}_{+} \times \mathbb{R}^{n} \times \mathbb{R}^{n}:\right. \\
& |x|>1, \quad x \cdot y>0, \quad t|x|>1, \quad t^{2}|x|<|x|-\left|y_{x}\right|<|x| / 4, \quad\left|y_{x}^{\prime}\right|<\sqrt{\left.\frac{|x|-\left|y_{x}\right|}{|x|}\right\},}
\end{aligned}
$$

and $P_{t}(x, y) \simeq K_{3}(t, x, y)$ uniformly in

$$
E_{3}=\left\{(t, x, y) \in \mathbb{R}_{+} \times \mathbb{R}^{n} \times \mathbb{R}^{n}: t>1,|x|<1,|y|<1\right\} .
$$

Finally, $P_{t}(x, y) \simeq K_{4}(t, x, y)$ uniformly in

$$
\begin{aligned}
E_{4}= & \left\{(t, x, y) \in \mathbb{R}_{+} \times \mathbb{R}^{n} \times \mathbb{R}^{n}:\right. \\
& \left.|x|>e^{16}, \quad t=\frac{\sqrt{\log |x|}}{2}, \quad|x|^{2 / 3} \leq\left|y_{x}\right| \leq|x|^{3 / 4}, \quad\left|y_{x}^{\prime}\right|<1\right\} .
\end{aligned}
$$


(b) In the estimate in Theorem 1.2, none of the terms $K_{i}(t, x, y), i=1,2,3,4$, can be suppressed.

Proof. To prove (a), we only need to consider $x=\left(x_{1}, 0, \ldots, 0\right)$ with $x_{1} \geq 0$ and write $y=$ $\left(y_{1}, y^{\prime}\right)$. We shall use several estimates from the proof of Proposition 4.1. Observe that points of $E_{1}$ and $E_{2}$ belong to (ii) of Proposition 4.1 and satisfy $t<1 / 2$.

Assume $(t, x, y) \in E_{1}$. Then

$$
x_{1}>1, \quad t^{2} x_{1}<x_{1}-y_{1}<x_{1}^{-1} / 4 \quad \text { and } \quad\left|y^{\prime}\right|<x_{1}-y_{1} .
$$

Transforming variables in the integral in (4.12), we get

$$
J_{1,1} \simeq \frac{t}{\left(t^{2}+|x-y|^{2}\right)^{(n+1) / 2}} \int_{0}^{B} \frac{1}{u^{(n+3) / 2}} \exp ^{*}\left(-\frac{1}{u}\right) d u,
$$

with $B=3\left(x_{1}-y_{1}\right) /\left(4 x_{1}\left(t^{2}+|x-y|^{2}\right)\right)$. One easily verifies that $B^{-1} \lesssim 1$, so that the value of the integral here stays away from 0 . Since also $t(1+|x|) \lesssim 1$, it follows that $J_{1,1} \simeq$ $t /\left(t^{2}+|x-y|^{2}\right)^{(n+1) / 2} \simeq K_{1}(t, x, y)$. Consequently, $P_{t}(x, y) \gtrsim K_{1}(t, x, y)$ in $E_{1}$.

To obtain the converse inequality, we notice that Proposition 4.1(ii) applies, and its proof shows that $P_{t}(x, y) \lesssim J_{1,1}+J_{1,2}+J_{1,3} \lesssim K_{1}(t, x, y)+J_{1,2}+K_{3}(t, x, y)$. The inequalities (4.14) now imply that $J_{1,2} \lesssim K_{1}(t, x, y)$, since $x_{1} /\left(x_{1}-y_{1}\right)<\left(x_{1}-y_{1}\right)^{-2}$ in $E_{1}$. Further,

$$
K_{3}(t, x, y) \simeq t \exp ^{*}\left(-|y|^{2}\right) \lesssim t \exp ^{*}\left(-|x|^{2}\right) \lesssim K_{1}(t, x, y) .
$$

We conclude that $P_{t}(x, y) \simeq K_{1}(t, x, y)$ in $E_{1}$.

Now assume $(t, x, y) \in E_{2}$ so that

$$
x_{1}>1, \quad t x_{1}>1, \quad t^{2} x_{1}<x_{1}-y_{1}<x_{1} / 4 \quad \text { and } \quad\left|y^{\prime}\right|<\sqrt{\left(x_{1}-y_{1}\right) / x_{1}} .
$$

Then $K_{2}(t, x, y) \simeq t x_{1}^{n / 2}\left(x_{1}-y_{1}\right)^{-(n+2) / 2}$. Since $x_{1}\left(x_{1}-y_{1}\right)>t^{2} x_{1}^{2}>1$, a simple scaling shows that the second integral in (4.13) has order of magnitude $\left(\left(x_{1}-y_{1}\right) / x_{1}^{3}\right)^{1 / 2}$. The exp* factor preceding it is essentially 1 , and we conclude that

$$
J_{1,2} \simeq t\left(\frac{x_{1}}{x_{1}-y_{1}}\right)^{\frac{n+3}{2}}\left(\frac{x_{1}-y_{1}}{x_{1}^{3}}\right)^{\frac{1}{2}} \simeq K_{2}(t, x, y) .
$$

Thus $P_{t}(x, y) \gtrsim K_{2}(t, x, y)$. In $E_{2}$ one also has $K_{1}(t, x, y) \lesssim t /\left(x_{1}-y_{1}\right)^{n+1}$ and $K_{3}(t, x, y) \lesssim$ $t \exp ^{*}\left(-x_{1}^{2}\right)$, and these quantities are controlled by $K_{2}(t, x, y)$. Proposition 4.1(ii) then shows that $P_{t}(x, y) \lesssim K_{2}(t, x, y)$. Thus $P_{t}(x, y) \simeq K_{2}(t, x, y)$ in $E_{2}$.

Assume next that $(t, x, y) \in E_{3}$ so that $K_{3}(t, x, y) \simeq 1$. Now (4.11) is sharp and leads to $J_{2} \simeq 1 \simeq K_{3}$. Also, $K_{2}(t, x, y)=K_{4}(t, x, y)=0$, and $K_{1}(t, x, y) \lesssim t^{-n} \lesssim 1$. It follows that $P_{t}(x, y) \simeq K_{3}(t, x, y)$ in $E_{3}$.

Finally let $(t, x, y) \in E_{4}$. Then the estimate (4.15) is sharp since $y_{1}>1$, and so $J_{2,2} \simeq$ $K_{4}(t, x, y)$. Further, one verifies that $K_{4}(t, x, y) \gtrsim x_{1}^{-3 / 4}\left(\log x_{1}\right)^{-1}$ and also that $K_{1}(t, x, y)$ and $K_{3}(t, x, y)$ are controlled by $\exp ^{*}\left(-x_{1}\right) \lesssim K_{4}(t, x, y)$. It now follows from Proposition 4.1(iii) that $P_{t}(x, y) \simeq K_{4}(t, x, y)$ in $E_{4}$. 
This completes the arguments for (a).

We prove (b) by finding for each $\varepsilon>0$ and $i=1,2,3,4$ a nonempty subset $\tilde{E}_{i}$ of $E_{i}$ in which $K_{j}<\varepsilon P_{t}$ for $j \neq i$. In the proof below, we fix $\varepsilon$ and denote by $C_{\varepsilon}$ various large positive constants which may depend on $\varepsilon$.

Let

$$
\widetilde{E}_{1}=\left\{(t, x, y) \in E_{1}:|x|>C_{\varepsilon}, \quad t=\frac{1}{|x|^{2}}, \frac{1}{|x|^{2}}<|x|-\left|y_{x}\right|<\frac{2}{|x|^{2}}\right\} .
$$

In this set, $P_{t}(t, x, y) \simeq K_{1}(t, x, y) \simeq|x|^{2 n}$ but $K_{2}(t, x, y) \simeq|x|^{3 n / 2}$ and $K_{3}(t, x, y) \lesssim 1$, whereas $K_{4}(t, x, y)$ vanishes. A suitable choice of $C_{\varepsilon}$ yields the desired inequalities.

In a similar way, we define

$$
\widetilde{E}_{2}=\left\{(t, x, y) \in E_{2}:|x|>C_{\varepsilon}, \quad t=|x|^{-1 / 2}, \quad 1<|x|-\left|y_{x}\right|<2\right\},
$$

and it is enough to observe that in this set $P_{t}(t, x, y) \simeq K_{2}(t, x, y) \simeq|x|^{(n-1) / 2}$, but $K_{1}(t, x, y) \lesssim$ $\exp ^{*}\left(-|x|^{1 / 2}\right)$ and $K_{3}(t, x, y) \lesssim \exp ^{*}\left(-|x|^{2}\right)$ and $K_{4}(t, x, y)=0$.

The next set is

$$
\widetilde{E}_{3}=\left\{(t, x, y) \in E_{3}: t>C_{\varepsilon}\right\},
$$

in which $P_{t}(t, x, y) \simeq K_{3}(t, x, y) \simeq 1$ but $K_{1}(t, x, y) \lesssim t^{-n}$ and $K_{2}(t, x, y)=K_{4}(t, x, y)=0$.

Finally,

$$
\widetilde{E}_{4}=\left\{(t, x, y) \in E_{4}:|x|>C_{\varepsilon}\right\} .
$$

To compare the kernels $K_{i}(t, x, y)$ on this set, it is enough to consider the last part of the proof of (a).

This ends the proof of (b) and that of the theorem.

\section{References}

[1] G. Garrigós, S. Harzstein, T. Signes, J. L. Torrea and B. Viviani, Pointwise convergence to intial data of heat and Laplace equations, Submitted.

[2] A. E. Gatto, E. Pineda, and W. Urbina, Riesz potentials, Bessel potentials, and fractional derivatives on Besov-Lipschitz spaces for the Gaussian measure, Recent Advances in Harmonic Analysis and Applications Springer Proceedings in Mathematics \& Statistics, Volume 25, 2013, pp 105-130.

[3] A. E. Gatto and W. Urbina, On Gaussian Lipschitz spaces and the boundedness of fractional integrals and fractional derivatives on them, arXiv:0911.3962.

[4] E. Pineda and W. Urbina, Some results on Gaussian Besov-Lipschitz spaces and Gaussian Triebel-Lizorkin spaces, J. Approx. Theory 161 (2009), 529-564.

[5] E. M. Stein, Singular Integrals and Differentiability Properties of Functions, Princeton University Press, Princeton, N.J. 1970 xiv+290 pp.

[6] E. M. Stein, Topics in Harmonic Analysis Related to the Littlewood-Paley Theory, Princeton University Press, Princeton, N.J.; University of Tokyo Press, Tokyo, 1970 viii+146 pp. 
LIGUANG LIU

Department of Mathematics, School of Information Renmin University of China

Beijing 100872

China

E-mail: liuliguang@ruc.edu.cn

PETER SJÖGREN

Mathematical Sciences, University of Gothenburg

and

Mathematical Sciences, Chalmers

SE-412 96 Göteborg

Sweden

E-mail: peters@chalmers.se 